\title{
Modeling of Elastic Modulus of CNT/Silicone Composites Designed for Medical Applications
}

\author{
M.N. Barshutina ${ }^{1} *$, P.E. Musienko ${ }^{1}$, A.G. Tkachev ${ }^{2}$ \\ ${ }^{1}$ Laboratory of Neuroprosthetics, Institute of Translational Biomedicine, Saint- Petersburg State University, \\ Universitetskaya Naberezhnaya 7-9, 199034, St. Petersburg, Russian Federation; \\ ${ }^{2}$ Department of Technology and Methods of Nanoproducts Manufacturing, Tambov State Technical University, \\ Leningradskaya ul., 1, 392000, Tambov, Russian Federation \\ * Corresponding author. Tel.: +7 92023188 85.E-mail: barshutina.marie@yandex.ru
}

\begin{abstract}
This paper deals with mathematical modeling of the elastic modulus of polymer composites based on carbon nanotubes. These composites are promising materials for electrodes of flexible medical implants due to their good conductivity, low elastic modulus and high biocompatibility. However, to achieve a high level of affinity of these composites to biological tissues, it is necessary to ensure the maximum possible overlapping of composite elastic moduli withelastic moduli of tissues. As a result of theoretical and experimental studies, we obtained the mathematical model that allows calculating the optimal concentrations of carbon nanotubes in composites to reach an elastic modulus of particular tissues.
\end{abstract}

\section{Keywords}

Nanocomposites; mathematical modeling; elastic modulus; carbon nanotubes; biological tissues; medical implants.

(c) M.N. Barshutina, P.E. Musienko, A.G. Tkachev, 2020

\section{Introduction}

Recently, composites based on carbon materials are becoming increasingly widespread in various branches of science and technology due to their outstanding, and sometimes even unique, properties. In particular, composites based on carbon nanotubes (CNT) have been proposed as the most promising material for neural implant electrode sowing to their sufficiently high conductivity and specific capacitance[1-3].

The most important advantages of CNT/polymer composites in front of traditionally used platinum $(\mathrm{Pt})$ and gold $(\mathrm{Au})$ are their low stiffness and high flexibility. The elastic modulus of such composites can fit even to the elastic modulus of the protective tissues of the nervous system (20 MPa) [4], while the elastic moduli of $\mathrm{Au}$ and $\mathrm{Pt}$ exceed it by several orders of magnitude and reach 79 and $173 \mathrm{GPa}$, respectively. Such a significant reduction in the elastic modulus of the material used to fabricate the electrodes of medical implants can significantly reduce the risk of damage to the surrounding tissue and its inflammatory response.
Moreover, the elastic modulus of $\mathrm{CNT} /$ polymer composites can be precisely adjusted to elastic moduli of particular tissues (Fig. 1) by varying the CNT concentration and type of binder polymer. The use of this approach will help facilitate the biocompatibility of medical implants and their affinity to biological tissues.

To create CNT/polymer composites with an elastic modulus of a particular tissue, it is necessary to develop a mathematical model that allows calculating the elastic modulus of composites from the concentrations of initial components. For this purpose, we carried out theoretical and experimental researches presented further.

\section{Theory}

There are several micromechanical models that allow calculating the elastic modulus of composites from the percentage ratio of their components. The most common are the following [6-9].

1. Voigt-Reuss model. This model allows calculating the lower and upper boundaries of the elastic modulus of composites that contain nanoobjects 


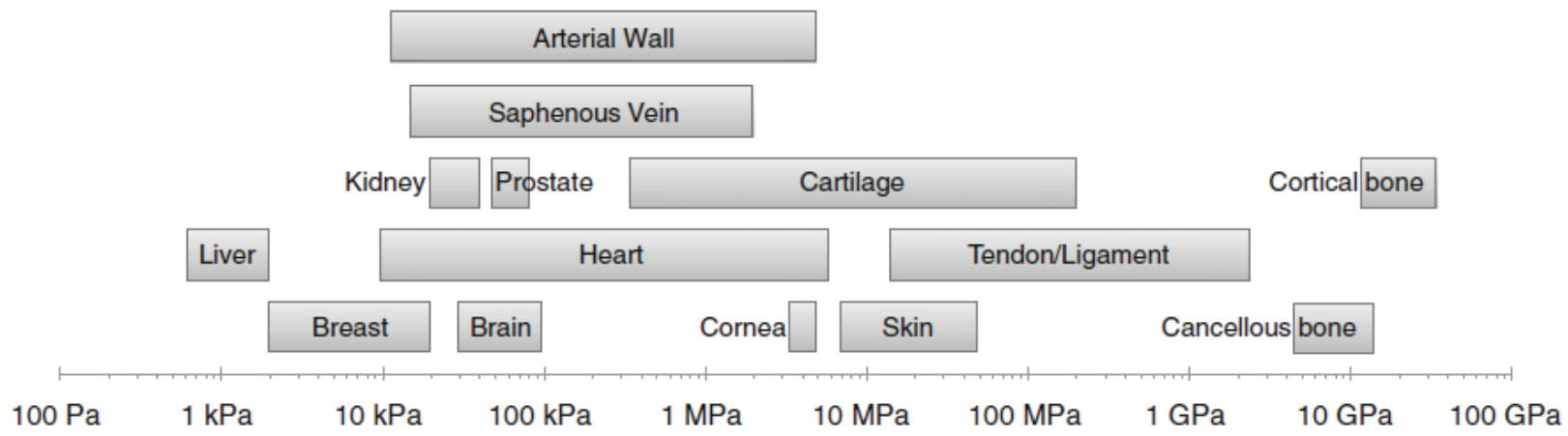

Fig. 1. Elastic moduli of different biological tissues [5]

elongated in one dimension (nanofibres or nanotubes). The upper limit of the elastic modulus is calculated by the Voigt formula:

$$
E_{||}=\varphi E_{\mathrm{f}}+(1-\varphi) E_{\mathrm{p}},
$$

where $\varphi$ is the volume fraction of the filler (nanofibres, nanotubes); $E_{\mathrm{f}}$ is the elastic modulus of the filler; $E_{\mathrm{p}}$ is the elastic modulus of the polymer.

The lower limit of the elastic modulus of nanostructured composites is calculated by the Reuss formula:

$$
1 / E_{\perp}=\varphi / E_{\mathrm{f}}+(1-\varphi) / E_{\mathrm{p}} .
$$

The values of elastic moduli $E_{\mid}$and $E_{\perp}$, calculated from formulae (1) and (2), correspond to the elastic moduli of composites that contain nanofibres or nanotubes oriented in one direction. In this case, $E_{||}$is the longitudinal elastic modulus measured parallel to the bedding plane of nanofibers or nanotubes, and $E_{\perp}$ is the transverse elastic modulus measured perpendicular to the bedding plane.

2. Hashin-Shtrikman model. This model is applicable to macroscopically isotropic composites and allows calculating the upper $E_{||}$and lower $E_{\perp}$ boundaries of the composite elastic modulus. The following formulas are used for this purpose:

$$
\begin{gathered}
K_{||}=K_{\mathrm{f}}+(1-\varphi)\left[\frac{1}{K_{\mathrm{p}}-K_{\mathrm{f}}}+\frac{3 \varphi}{3 K_{\mathrm{f}}+4 G_{\mathrm{f}}}\right]^{-1} ; \\
K_{\perp}=K_{\mathrm{p}}+\varphi\left[\frac{1}{K_{\mathrm{f}}-K_{\mathrm{p}}}+\frac{3(1-\varphi)}{3 K_{\mathrm{p}}+4 G_{\mathrm{p}}}\right]^{-1} ; \\
G_{||}=G_{\mathrm{f}}+(1-\varphi)\left[\frac{1}{G_{\mathrm{p}}-G_{\mathrm{f}}}+\frac{6 \varphi\left(K_{\mathrm{f}}+2 G_{\mathrm{f}}\right)}{5 G_{\mathrm{f}}\left(3 K_{\mathrm{f}}+4 G_{\mathrm{f}}\right)}\right]^{-1} ;
\end{gathered}
$$

$$
\begin{gathered}
G_{\perp}=G_{\mathrm{p}}+\varphi\left[\frac{1}{G_{\mathrm{f}}-G_{\mathrm{p}}}+\frac{6(1-\varphi)\left(K_{\mathrm{p}}+2 G_{\mathrm{p}}\right)}{5 G_{\mathrm{p}}\left(3 K_{\mathrm{p}}+4 G_{\mathrm{p}}\right)}\right]^{-1} ; \\
E_{||}=\frac{9 K_{||}}{1+3 K_{||} / G_{\mid}} ; \\
E_{\perp}=\frac{9 K_{\perp}}{1+3 K_{\perp} / G_{\perp}}
\end{gathered}
$$

where $K_{||}$is the upper limit of the bulk elastic modulus; $K_{\perp}$ is the lower limit of the bulk elastic modulus; $G_{\mid}$is the upper limit of the shear modulus; $G_{\perp}$ is the lower limit of the shear modulus; $K_{\mathrm{f}}$ is thebulkelastic modulus of the filler; $K_{\mathrm{p}}$ is bulk elastic modulus of the polymer; $G_{\mathrm{f}}$ is the shear modulus of the filler; $G_{\mathrm{p}}$ is the shear modulus of the polymer; $\varphi$ is the volume fraction of the filler in the composite.

3. Halpin-Tsai model. This model is a semiempirical model that applicable to composites with filler particles oriented in one direction (nanofibers, nanotubes, etc.). In this case, the longitudinal and transverse elastic moduli are calculated by the formulas:

$$
\begin{gathered}
E_{||}=\frac{1+2(l / d) \varphi \eta_{\mid} \mid}{1-\varphi \eta_{\mid}} E_{\mathrm{p}} ; \\
E_{\perp}=\frac{1+2 \varphi \eta_{\perp}}{1-\varphi \eta_{\perp}} E_{\mathrm{p}}
\end{gathered}
$$

where $l$ is the length of nanofibers or nanotubes; $d$ is the diameter of nanofibers or nanotubes; $\eta_{\mid}$and $\eta_{\perp}$ are coefficients given by: 


$$
\begin{gathered}
\eta_{\mid l}=\frac{E_{\mathrm{f}}-E_{\mathrm{p}}}{E_{\mathrm{f}}+2(l / d) E_{\mathrm{p}}} ; \\
\eta_{\perp}=\frac{E_{\mathrm{f}}-E_{\mathrm{p}}}{E_{\mathrm{f}}+2 E_{\mathrm{p}}} .
\end{gathered}
$$

Finally, it should be pointed out that Halpin-Tsai, Hashin-Shtrikman and Voigt-Reuss models can be used to calculate the elastic modulus of composites with randomly oriented filler nanoparticles. In this case, the values of $E_{||}$and $E_{\perp}$ calculated from Eqs. (1)-(2), (7)-(8) and (9)-(10) are substituted into the equation [10]:

$$
E_{\mathrm{c}}=3 E_{||} / 8+5 E_{\perp} / 8 .
$$

\section{Materials and methods}

We calculated the elastic moduli of composites with CNT mass fractions 1, 3, 5, 7 and $9 \%$ by applying the Voigt-Reuss, Hashin-Shtrikman and Halpin-Tsai models. Wherein the elastic moduli $(E)$, shear moduli $(G)$ and bulk elastic moduli $(K)$ of pure silicone and carbon nanotubes $[11,12]$ were taken as values of $E_{\mathrm{p}}$, $E_{\mathrm{f}}, G_{\mathrm{p}}, G_{\mathrm{f}}, K_{\mathrm{p}}$ and $K_{\mathrm{f}}$.

Further, to carry out the experimental investigations, we prepared samples of composites with different concentrations of carbon nanotubes «Taunit» (NanoTechCenter, Tambov, Russia). The 2-component 10: 1, optically transparent, dipping silicone compound Dow Corning Sylgard 184 (Midland, USA) was used as a polymer base. The CNTs were added to the fluid base of the silicone compound in a mass quantity of $1,3,5$, 7 and $9 \%$. To achieve an even distribution of CNTs in a polymer base, all the mixtures were alternately placed in a three-roll mill (EXAKT 80E, EXAKT Technologies, Inc) for 15 min. Further, the obtained homogeneous substances were mixed with the curing agent, filled into metal molds and placed in a vacuum oven (VTSH-K52-250, OOO AKTAN VACUUM) at a temperature of $125^{\circ} \mathrm{C}$ for $20 \mathrm{~min}$.

The elastic moduli of obtained samples and micrographs of their surfaces were defined by PI87 SEM PicoIndenter (Hysitron Inc.) and Zeiss Merlin FE-SEM (Carl Zeiss Microscopy LLC).

\section{Results and discussion}

Elastic moduli of $\mathrm{CNT} /$ silicone composites obtained from theoretical and experimental investigations are presented in Fig. 2.

According to the obtained results, Voigt-Raus and Hashin-Shtrikman models are not applicable to CNT/silicone composites since elastic moduli calculated from these models are 1000 times higher than moduli obtained experimentally. Also we can conclude that the most appropriate theoretical model for characterization of $\mathrm{CNT} /$ silicone composites is the Halpin-Tsai model since elastic moduli calculated from this model are comparable with experimental data. Moreover, experimental data and data obtained from Halpin-Tsai model can be approximated (with approximation error $<2 \%$ ) by linear functions (Fig. 3):

$$
\begin{gathered}
E_{\mathrm{HT}}=103 \varphi+2.46 ; \\
E_{\text {exp }}=52 \varphi+2.46 .
\end{gathered}
$$

The slope mismatch of the approximating functions indicates that the nanotubes in the experimental samples are oriented not in a random way,

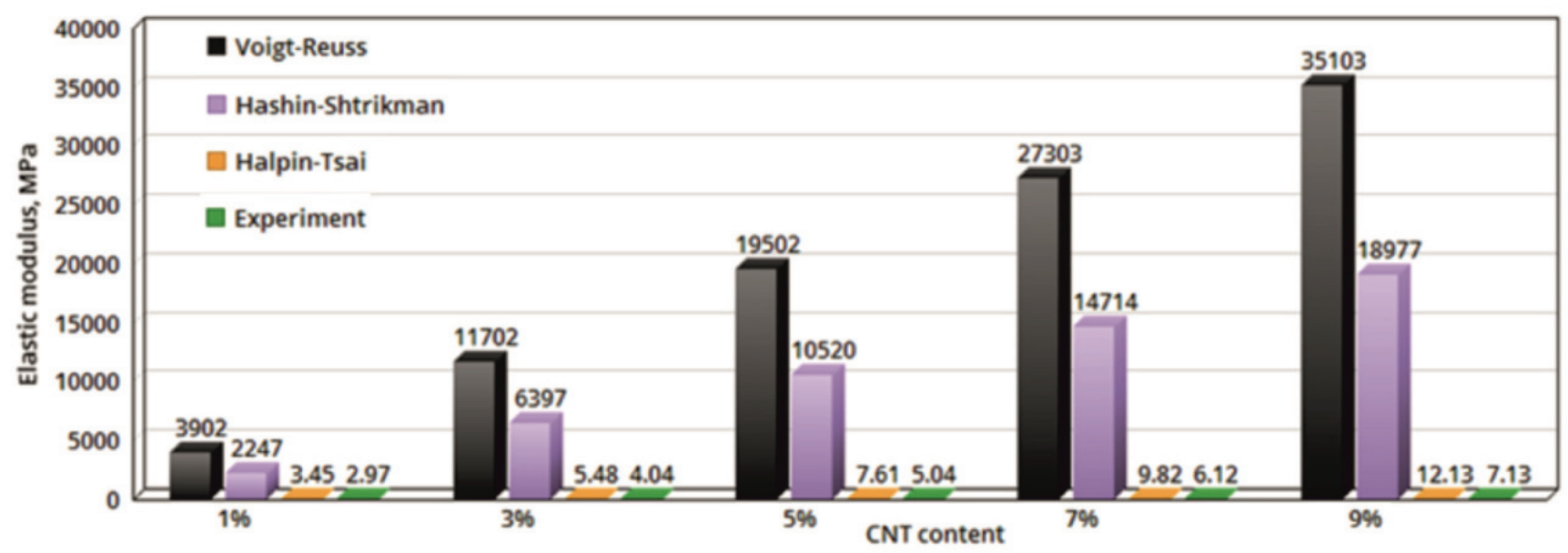

Fig. 2. Composite elastic moduli obtained experimentally and calculated from models 


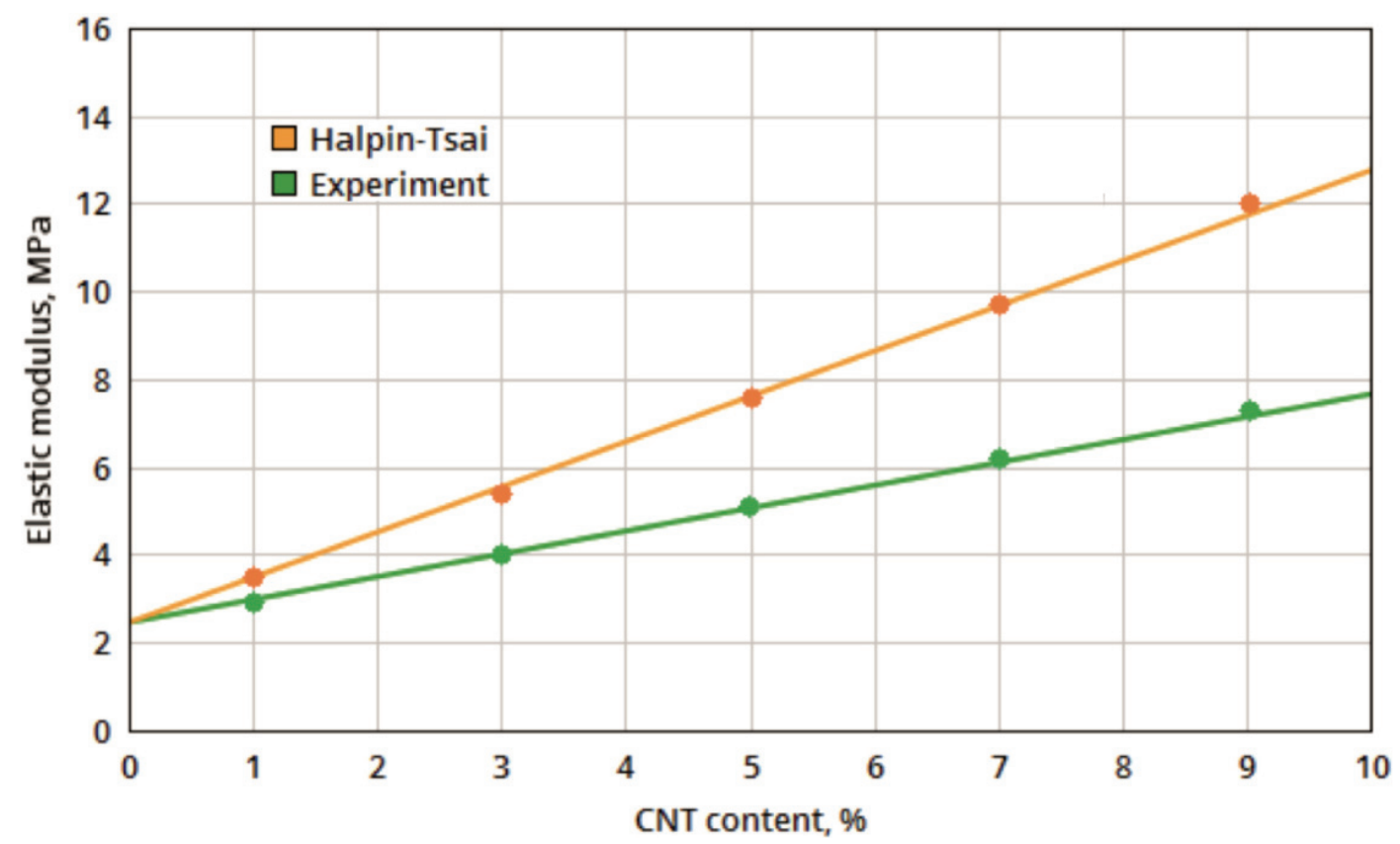

Fig. 3. Functions approximating elastic moduli obtained from Halpin-Tsai model and experiments

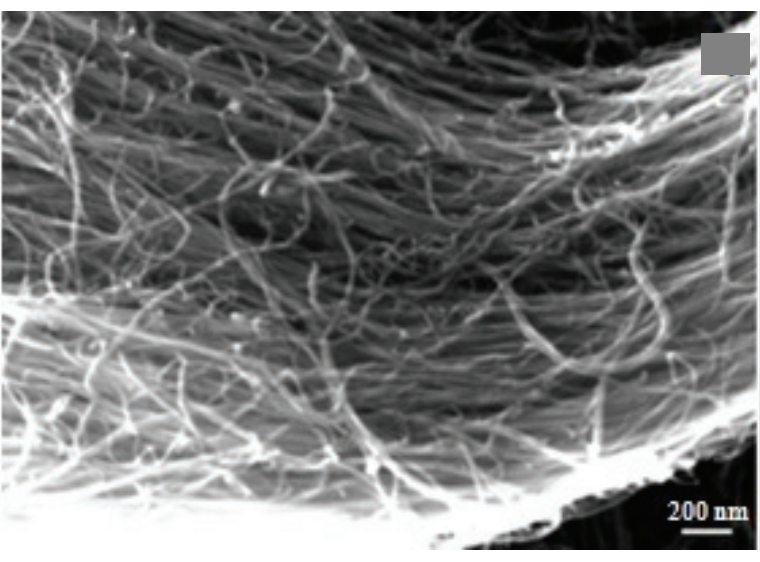

a)

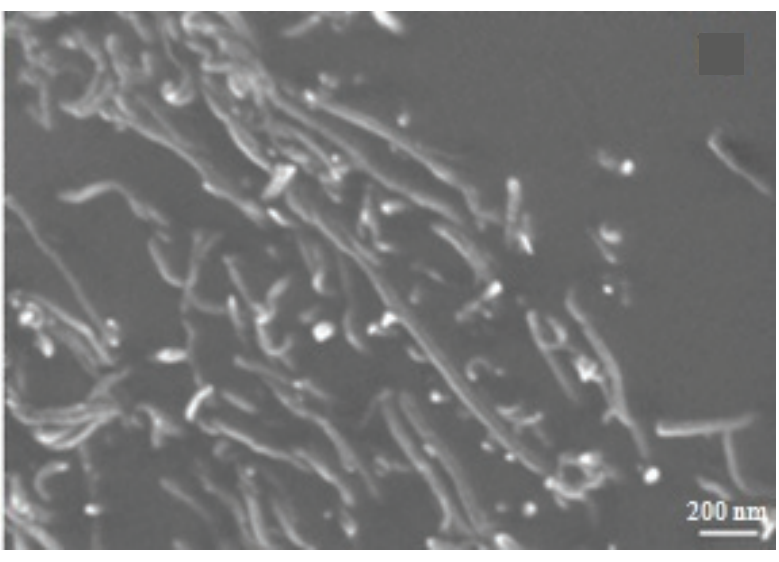

b)

Fig. 4. The SEM image of pristine CNT (a), surface of CNT/silicone composite (b)

but mainly in one direction. By varying the longitudinal and transverse components in Eq. (13), we found that the maximum correspondence of the experimental and theoretical data is achieved if the ratio of longitudinal and transverse components is $1.35: 6.65$. Consequently, following formula should beused instead of Eq. (13):

$$
E_{\mathrm{c}}=1.35 E_{||} / 8+6.65 E_{\perp} / 8 .
$$

The ratio of the longitudinal and transverse components indicates that in the composite samples the fraction of nanotubes oriented in one direction is about $75 \%$, and these results are in accordance with the results obtained from SEM (Fig. 4).

\section{Conclusions}

As a result of theoretical and experimental studies, it was found that the elastic modulus of composites based on CNT "Taunit" can be described by HalpinTsai model with the correction coefficients considering theorientation of nanotubes in composites. In this work the correction coefficients were found experimentally for $\mathrm{CNT} /$ silicone composites, and their values were used to predict the ratio of longitudinally and transversely oriented nanotubes. Also we obtained the approximating functions that allow calculating the elastic modulus of composites from concentrations of initial components. On the other hand, the same equations can be used to find out the optimal 
concentrations of carbon nanotubes in polymer composites to achieve the elastic modulus of particular biologicaltissues. This solution has a great importance for engineering of new materials applied in medical implantation. The adjustment of elastic moduli of implant surfaces to elastic moduli of surrounding tissues will help facilitate the biocompatibility of medical implants, and significantly enhance their affinity to particular biological tissues.

\section{References}

1. Tegtmeier K., Aliuos P., Lenarz T. et al. Residual rubber shielded multi walled carbon nanotube electrodes for neural interfacing in active medical implants, Physics in Medicine, 2016, 1 8-19.

2. Stieghorst J., Bondarenkova A., Burblies N. et al. 3D silicone rubber interfaces for individually tailored implants, Biomed Microdevices, 2015, 17, 54

3. Brensing A., Ruff R., Fischer B. et al. PDMS electrodes for recording and stimulation, Current Directions in Biomedical Engineering, 2017, 3(1), 63-67.

4. Karnaushenko D., Münzenrieder N., Karnaushenko D.D. et al. Biomimetic Microelectronics for Regenerative Neuronal Cuff Implants, Adv. Mater., 2015, 27, 6797-6805.

5. Kim H.N., Kang D.H., Kimet M.S. et al. Patterning methods for polymers in cell and tissue engineering, Annals of Biomedical Engineering, 2012, 40(6), 1339-1355.
6. Gholap S., Panchagade D.R., Patil V. Continuum modeling techniques to determine mechanical properties of nanocomposites, International Journal of Modern Engineering Research, 2014, 4(1), 9-15.

7. Voigt W. Ueber die Beziehung zwischen den beiden Elasticitätsconstanten isotroper Körper, Annalen der Physik, 1889, 274, 573-587.

8. Reuss A. Berechnung der Fließgrenze von Mischkristallen auf Grund der Plastizität sbedingung für Einkristalle, Zeitschriftfür Angewandte Mathematik und Mechanik, 1929, 9, 49-58.

9. Halpin J.C., Kardos J.L. Halpin-Tsai equations: a review, Polymer Engineering and Science, 1976, 16, 344-352.

10. Tsai S.W., Halpin J.C. and Pangano N.J. (Eds.) Composite Materials Workshop, Technomic Publ. Co., Inc., Lancaster, 1968.

11. Johnston I.D., McCluskey D.K., Tan C.K.L., Tracey M.C. Mechanical characterization of bulk Sylgard 184 for microfluidics and microengineering, $J$ Micromech Microeng, 2014, 24(3), 035017.

12. Milowska K.Z. Influence of Carboxylation on Structural and Mechanical Properties of Carbon Nanotubes: Composite Reinforcement and Toxicity Reduction Perspectives, Journal of Physical Chemistry C, 2015, 119(47), 26734-26746.

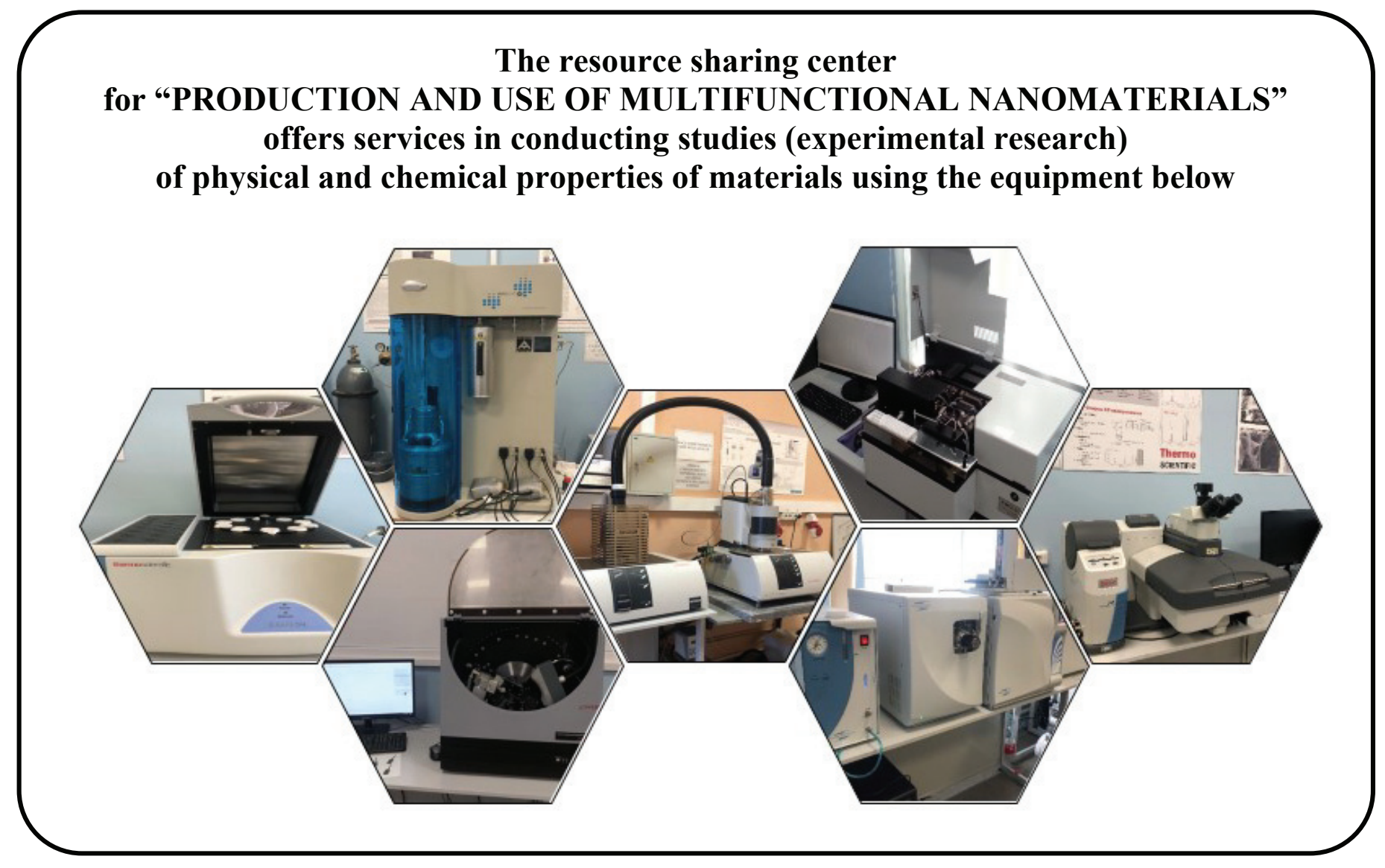

